\title{
BOREL MEASURABLE IMAGES OF POLISH SPACES
}

\author{
SANDRO LEVI AND ASHOK MAITRA
}

\begin{abstract}
We determine the Borel class of the image of a Polish space under a Borel measurable function of class $\eta$ which maps open sets in the Polish space to Borel sets of additive class $\xi$ in the range, under a mild restriction on the inverse images of singleton sets. Our computation is based on a selection theorem proved in this article.
\end{abstract}

1. Introduction. Our point of departure in this article is a result of Srivastava [8] which states the following: if $Z$ is a Polish space, $X$ a separable metric sp: ce and $f$ a Borel measurable function of class 1 on $Z$ onto $X$ such that $f$ maps upen sets of $Z$ to Borel sets in $X$, then $X$ is an absolute Borel set (that is, a Borel set in its completion). Suppose now that in fact $f$ maps open sets of $Z$ to Borel sets of additive class $\xi$ in $X$. The present article is the result of our efforts to determine the Borel class of $X$ in its completion. It turns out that if $\xi>0$ then $X$ is of multiplicative class $\xi+1$ in its completion. Our proof of the result rests on a refinement of a selection theorem of Burgess [1]; the refinement consists of an evaluation of the Borel class of the selector. Indeed, using our selection theorem, we are able to evaluate the Borel class of the image of a Polish space under a Borel measurable function of class $\eta$ which maps open sets in the Polish space to Borel sets of additive class $\xi$ in the range, provided that the inverse image of each singleton set is comeager in its closure. This result in turn yields a theorem of Miller [7] about the Borel class of a selector for a partition of a Polish space into $G_{\delta}$ sets.

The paper is organized as follows. $\S 2$ is devoted to definitions and notation. In $\S 3$ we prove the selection theorem, and in $\S 4$ we evaluate the Borel class of the image of a Polish space under a function of the type described in the previous paragraph. Miller's theorem on measurable selectors is deduced in $\S 5$.

2. Definitions and notation. Our usage regarding Borel classes of sets and functions is standard and can be found in Kuratowski [4]. We drop the appellation "Borel" when referring to a Borel function of a particular class or a Borel set of a particular class. Thus we write "function of class $\xi$ " and "set of additive class $\xi$ " etc. If $Z, X$ are metric spaces, a function $f$ on $Z$ onto $X$ is said to be a $(\eta, \xi)$ function if the inverse image of each open set in $X$ is a set of additive class $\eta$ in $Z$ (that is, $f$ is a function of class $\eta$ ) and the image of each open set in $Z$ is of additive class $\xi$ in $X$. Thus a continuous, open function on $Z$ onto $X$ is a $(0,0)$-function. If a $(\eta, \xi)$-function $f$ is one-one, we shall call $f$ a homeomorphism of class $\eta, \xi$ in conformity with [4].

Received by the editors September 6, 1983.

1980 Mathematics Subject Classification. Primary 54C65, 28B20; Secondary 28A05, 54H05.

Key words and phrases. Polish space, Borel class of sets and functions, selectors. 
A multifunction $F$ on $Z$ to $X$ is a function that takes points of $Z$ to nonempty subsets of $X$. If $F$ is a multifunction on $Z$ to $X$, the set $\{(z, x) \in Z \times X: x \in F(z)\}$ is called the graph of $F$ and denoted by $\operatorname{Gr}(F)$. If $V$ is a subset of $X$, the set $\{z \in Z: F(z) \cap V \neq \emptyset\}$ will be denoted by $F^{-1}(V)$. The multifunction $F$ is said to be of class $\xi$ if $F^{-1}(V)$ is of additive class $\xi$ in $Z$ for every open set $V$ in $X$. The multifunction $z \rightarrow \operatorname{cl}(F(z))$ is denoted by $\bar{F}$, where cl abbreviates closure. A selector for $F$ is a function $f: Z \rightarrow X$ such that $f(z) \in F(z)$ for each $z \in Z$.

If $\Pi$ is a partition of a metric space $Z$ and $V \subseteq Z$, the set $\bigcup\{P \in \Pi: P \cap V \neq \emptyset\}$ is called the $\Pi$-saturation of $V$ and denoted by $V^{*}$. A selector for $\Pi$ is a function $f: Z \rightarrow Z$ such that $f$ is constant on each member of $\Pi$ and $f(P) \subseteq P$ for each $P \in \Pi$. A transversal for $\Pi$ is a set $S \subseteq Z$ such that $S$ meets each member of $\Pi$ in exactly one point.

If $\eta$ is an ordinal greater than 0 , we set $\bar{\eta}=\xi$ if $\eta=\xi+1$, and $\bar{\eta}=\eta$ if $\eta$ is a limit ordinal.

3. A selection theorem. In this section we prove a selection theorem which will play a key role in the sequel. We need the following computation.

LEMMA 1. Let $X$ be a separable metric space, $Z$ a Polish space and $F$ a multifunction of class $\xi$ on $X$ to $Z$. For each $W \subseteq X \times Z$, let $W^{+}=\left\{x \in X: W_{x} \cap \bar{F}(x)\right.$ is nonmeager in $\bar{F}(x)\}$, where $W_{x}$ is the $x$-section of $W$. If $W$ is of additive class $\eta$ in $X \times Z$, then $W^{+}$is of additive class $\xi+\eta$ in $X$.

PROOF. We prove the lemma by induction on $\eta$. Suppose first that $\eta=0$, so that $W$ is open in $X \times Z$. Hence we may write $W=\bigcup_{n \geq 0} C_{n} \times D_{n}$, where $C_{n}$ is open in $X$ and $D_{n}$ open in $Z$. Since, for each $x \in X, \bar{F}(x)$ is Polish, it follows by the Baire category theorem that

$$
x \in W^{+} \leftrightarrow W_{x} \cap \bar{F}(x) \neq \emptyset \leftrightarrow(\exists n)\left(x \in C_{n} \cap F^{* 1}\left(D_{n}\right)\right)
$$

so that $W^{+}=\bigcup_{n \geq 0} C_{n} \cap F^{-1}\left(D_{n}\right)$. Consequently $W^{+}$is of additive class $\xi$.

Suppose now that $\eta>0$ and that the lemma is true for all subsets of $X \times Z$ of additive class $\zeta<\eta$. Fix $\zeta<\eta$ and let $W$ be a set of multiplicative class $\zeta$ in $X \times Z$. For each $x \in X, W_{x} \cap \bar{F}(x)$ is a Borel subset of the Polish space $\bar{F}(x)$, and hence $W_{x} \cap \bar{F}(x)$ has the Baire property in $\bar{F}(x)$. Consequently, if $V_{n}, n \geq 0$, is a countable base for the topology of $Z$, then

$$
\begin{aligned}
x \in W^{+} & \leftrightarrow(\exists n)\left(x \in F^{-1}\left(V_{n}\right) \& W_{x}^{c} \cap V_{n} \cap \bar{F}(x) \text { is meager in } \bar{F}(x)\right) \\
& \leftrightarrow(\exists n)\left(x \in F^{-1}\left(V_{n}\right) \& x \notin\left(W^{c} \cap\left(X \times V_{n}\right)\right)^{+}\right),
\end{aligned}
$$

hence

$$
W^{+}=\bigcup_{n \geq 0}\left[F^{-1}\left(V_{n}\right) \cap\left(X \backslash\left(W^{c} \cap\left(X \times V_{n}\right)\right)^{+}\right)\right] .
$$

Now $W^{c} \cap\left(X \times V_{n}\right)$ is a set of additive class $\zeta$ in $X \times Z$, so by induction hypothesis $\left(W^{c} \cap\left(X \times V_{n}\right)\right)^{+}$is of additive class $\xi+\zeta$ in $X$. Hence the set within square brackets is of additive class $\xi+\zeta+1$, so $W^{+}$is of additive class $\xi+\varsigma+1$ in $X$.

Finally, let $W$ be of additive class $\eta$ in $X \times Z$. So there exist sets $W_{n}$ of multiplicative class $\eta_{n}(<\eta)$ in $X \times Z$ such that $W=\bigcup_{n \geq 0} W_{n}$. But then $W^{+}=\bigcup_{n \geq 0} W_{n}^{+}$. By the preceding paragraph, $W_{n}^{+}$is of additive class $\xi+\eta_{n}+1(\leq \xi+\eta)$ in $X$, so $W^{+}$is of additive class $\xi+\eta$ in $X$. This completes the proof. 
The special case of the above lemma when $F(x) \equiv Z$ was proved by Vaught [9]. The above proof closely follows Vaught's.

The selection theorem can now be proved.

THEOREM 1. Let $X$ be a separable metric space and $Z$ a Polish space. Suppose $F$ is a multifunction of class $\xi$ on $X$ to $Z$ such that $\operatorname{Gr}(F)$ is of multiplicative class $\eta+1$ in $X \times Z$ and $F(x)$ is comeager in $\bar{F}(x)$ for each $x \in X$. If $\xi+\eta>0$ (or if $\xi=0=\eta$ and $X$ is 0-dimensional), then there is a selector for $F$ of class $\xi+\eta$.

ProOF. Let $\tilde{X}$ be the completion of $X$ and $\tilde{B}$ be a set of multiplicative class $\eta+1$ in $\tilde{X} \times Z$ such that $\operatorname{Gr}(F)=\tilde{B} \cap(X \times Z)$. By a known result there is a $(0, \eta)$-function $g$ on $\omega^{\omega}$, the space of irrationals, onto $\tilde{B}$.

At this point we follow the proof of the theorem in [6], refining it as we go along. We also use the notation of [6]. First specialize the theorem of $[6]$ to the case where $J_{x}=\{E \subseteq \bar{F}(x): E$ is a meager Borel set in $\bar{F}(x)\}$. Note that the only properties of $g$ used in the proof in [6] are that $g$ is continuous and that $g(N(s)$ ) is Borel in $\tilde{B}$. The refinement that is needed here is to observe that when the hypotheses of our present theorem are in force we can define the sets $T(s, t)$ to be of additive class $\xi+\eta$ in $X$ instead of just being Borel in $X$ as was done in [6]. To see that this can be achieved, we note that $g(N(s)) \cap(X \times V(t))$ is of additive class $\eta$ in $\operatorname{Gr}(F)$. So we can find a set $W$ of additive class $\eta$ in $X \times Z$ such that $g(N(s)) \cap(X \times V(t))=W \cap \operatorname{Gr}(F)$. It follows that

$$
W^{+}=\left\{x \in X:(g(N(s)) \cap(X \times V(t)))_{x} \notin J_{x}\right\}
$$

since $\bar{F}(x) \backslash F(x)$ is meager in $\bar{F}(x)$ for each $x \in X$. Hence, in the notation of [6], the sets $(g(N(s)) \cap(X \times Y))^{* V(t)}$ are of additive class $\xi+\eta$ in $X$ by virtue of Lemma 1 of the present paper. We can now proceed to define the sets $T(s, t)$ as in [6]. For the inductive step we define the set $T^{1}(m, n)$ as in [6], noting here that the sets $T^{1}(m, n)$ are of additive class $\xi+\eta$ in $X$. The disjointification of the sets $T^{1}(m, n)$ is achieved by appealing to the Reduction Principle for sets of additive class $\xi+\eta([4$, p. 350] when $\xi+\eta>0 ;[4$, p. 279], when $\xi=0=\eta$ and $X$ is 0 -dimensional). This yields sets $T^{11}(m, n)$ of additive class $\xi+\eta$ in $X$, and we set $T(s m, t n)=T^{11}(m, n)$. The rest of the proof is just as in [6], yielding a selector $f: X \rightarrow Z$ for $F$ of class $\xi+\eta$. This completes the proof.

The selection theorem extends a result of Miller [7] in several directions.

4. Images of $(\eta, \xi)$-functions. We now use the selection theorem to determine the Borel class of the image of a Polish space under a $(\eta, \xi)$-function. The main result of this section follows.

THEOREM 2. Let $f$ be $a(\eta, \xi)$-function on a Polish space $Z$ onto a separable metric space $X$ such that $f^{-1}(\{x\})$ is comeager in $\operatorname{cl}\left(f^{-1}\{x\}\right)$ for each $x \in X$. If $\eta \geq 1$ and $\xi+\bar{\eta}>0$ (or if $\eta=1, \xi=0$ and $X$ is 0 -dimensional) then

(i) there is a function $g: X \rightarrow Z$ of class $\xi+\bar{\eta}$ such that $f(g(x))=x$ for each $x \in X$, and

(ii) $X$ is of multiplicative class $\xi+\bar{\eta}+\eta$ in its completion.

Proof. Define a multifunction $F$ on $X$ to $Z$ by setting $F(x)=f^{-1}(\{x\})$. Then $F$ is of class $\xi, \operatorname{Gr}(F)$ is of multiplicative class $\bar{\eta}+1[\mathbf{4}$, p. 384] and $F(x)$ is comeager 
in $\bar{F}(x)$ for each $x \in X$. So Theorem 1 applies to give a selector $g$ for $F$ of class $\xi+\bar{\eta}$. The function $g$ obviously satisfies the relation $f(g(x))=x$ for each $x \in X$.

To prove the second statement, use an extension theorem $[4$, p. 434] to find a set $X^{*}$ of multiplicative class $\xi+\bar{\eta}+1$ in $\tilde{X}$, the completion of $X$, and a function $\tilde{g}: X^{*} \rightarrow Z$ of class $\xi+\bar{\eta}$ such that $\tilde{g}=g$ on $X$. It follows that $X=\{x \in$ $\left.X^{*}: f(\tilde{g}(x))=x\right\}$, so that if $V_{n}, n \geq 0$, is a countable base for the topology of $X^{*}$, we have

$$
X^{*} \backslash X=\bigcup_{n \geq 0}\left[\tilde{g}^{-1}\left(f^{-1}\left(V_{n}\right)\right) \cap\left(X^{*} \backslash V_{n}\right)\right],
$$

so $X^{*} \backslash X$ is of additive class $\xi+\bar{\eta}+\eta$ in $X^{*}[4$, p. 376]. Hence $X$ is of multiplicative class $\xi+\bar{\eta}+\eta$ in $X^{*}$, so of multiplicative class $\xi+\bar{\eta}+\eta$ in $\tilde{X}$. This completes the proof.

Recalling that the inverse image of a singleton set under a function of class 1 is a $G_{\delta}$ and that each $G_{\delta}$ set is comeager in its closure, we get as an immediate consequence of Theorem 2 the following:

COROLlaRY. Let $f$ be a $(1, \xi)$-function on a Polish space $Z$ onto a separable metric space $X$. If $\xi>0$ (or if $\xi=0$ and $X$ is 0-dimensional), then $X$ is of multiplicative class $\xi+1$ in its completion.

We conclude this section with a couple of remarks. First, the assumption in Theorem 2 that $f^{-1}(\{x\})$ is comeager in $\operatorname{cl}\left(f^{-1}(\{x\})\right)$ for each $x \in X$ cannot be dropped. Indeed, there is a $(2,0)$-function on a Polish space onto an analytic nonBorel set. To see this, fix an analytic non-Borel subset $A$ of $\omega^{\omega}$, the space of irrationals, such that $A \subseteq N(0)$, the set of elements of $\omega^{\omega}$ that start with 0 . By an old result of Keldysh [3], there is a $F_{\sigma}$ subset $B$ of $\omega^{\omega} \times \omega^{\omega}$ such that $\Pi$, the projection to the first coordinate, when restricted to $B$ is an open mapping onto $A$. Let $\phi$ be a homeomorphism of $\left(\omega^{\omega} \times \omega^{\omega}\right) \backslash B$ and a closed subset $D$ of $N(1)$, the set of elements of $\omega^{\omega}$ that start with 1 . Define a function $f: \omega^{\omega} \times \omega^{\omega} \rightarrow \omega^{\omega}$ by setting $f=\Pi$ on $B$ and $f=\phi$ on $\left(\omega^{\omega} \times \omega^{\omega}\right) \backslash B$. Then $f$ is a $(2,0)$-function on $\omega^{\omega} \times \omega^{\omega}$ onto $A \cup D$, which is analytic non-Borel.

Second, the result of the Corollary is sharp. For if $X$ is separable metric of multiplicative class $\xi+1$ in its completion, then there is a homeomorphism of class $0, \xi$ on a Polish space onto $X[4$, p. 450]. When $\xi=0$ and $X$ is a separable metric space, not necessarily 0-dimensional, our corollary implies that $X$ is a $F_{\sigma \delta}$ in its completion. However, the following argument, due to S. M. Srivastava, shows that $X$ must be a $G_{\delta}$ in its completion. For if $\tilde{X}$ is the completion of $X$ and $G$ the graph of the function $f$, then $G$ is a $G_{\delta}$ in $Z \times \tilde{X}$. Moreover, as $f$ is open, if $\pi$ is the projection to the second coordinate, then $\pi$ restricted to $G$ is an open function from $G$ onto $X$. Consequently, $X$ is a continuous, open image of a $G_{\delta}$ subset of a Polish space, and so, by a well-known result of Hausdorff [2], $X$ is a $G_{\delta}$ in $\tilde{X}$.

5. Partitions of Polish spaces. In this section we shall use Theorem 2 to deduce a result of Miller regarding the Borel class of selectors and transversals for a partition of a Polish space.

We begin with a lemma of Miller [7], where the interested reader will find a proof. 
Lemma 2. Suppose $\Pi$ is a partition of a Polish space $Z$ into sets which are comeager in their closures. Let $V_{n}, n \geq 0$, be a countable base for the topology of $Z$. Then the sets $V_{n}^{*}, n \geq 0$, separate the members of $\Pi$.

What follows is Miller's result on selectors. Miller actually assumes that the members of the partition $\Pi$ are Baire spaces, besides assuming the same condition as in Theorem 3 below on the $\Pi$-saturations $V_{n}^{*}$ of the basic open sets $V_{n}, n \geq 0$. Now, according to Lemma 3.3 in [7], the first condition implies that the members of $\Pi$ are separated by $V_{n}^{*}, n \geq 0$. Consequently, in virtue of the second condition, each member of $\Pi$ is Borel in $Z$. But for a Borel subset $A$ of a Polish space $X$, it can be proved, (see [5]), that $A$ is a Baire space iff $A$ is comeager in $\operatorname{cl}(A)$. It is thus seen that Miller's hypotheses are equivalent to ours.

THEOREM 3. Let $Z$ be a Polish space and $\Pi$ a partition of $Z$ into sets which are comeager in their closures. Let $V_{n}, n \geq 0$, be a countable base for the topology of $Z$ and assume that for each $n$ the $\Pi$-saturation $V_{n}^{*}$ of $V_{n}$ is of ambiguous class $\eta>0$. Then there exist a selector for $\Pi$ of class $\eta+\bar{\eta}$ and a transversal for $\Pi$ of multiplicative class $\eta+\bar{\eta}$ in $Z$.

Proof. Define a function $f: Z \rightarrow 2^{\omega}$, the Cantor set, by

$$
f(z)=\left(I_{V_{1}^{*}}(z), I_{V_{2}^{*}}(z), \ldots\right)
$$

where $I_{V_{i}^{*}}$ is the characteristic function of $V_{i}^{*}$. Let $X=f(Z)$. Since $X \subseteq 2^{\omega}$, $X$ is 0 -dimensional. Furthermore, it is easy to see that $f$ is a $(\eta, 0)$-function on $Z$ onto $X$. Next observe that, by virtue of Lemma 2 , the sets of constancy of $f$ are precisely the members of $\Pi$, so that $f^{-1}(\{x\})$ is comeager in $\operatorname{cl}\left(f^{-1}(\{x\})\right)$ for each $x \in X$. Hence by Theorem 2 there is a function $g: X \rightarrow Z$ of class $\bar{\eta}$ such that $f(g(x))=x$ for each $x \in X$. Then the function $h=g \circ f$ is a selector for $\Pi$ of class $\eta+\bar{\eta}$. Moreover the set $S=\{z \in Z: h(z)=z\}$ is a transversal for $\Pi$ of multiplicative class $\eta+\bar{\eta}$ in $Z$, because $Z \backslash S=\bigcup_{n \geq 0}\left[h^{-1}\left(V_{n}\right) \cap\left(Z \backslash V_{n}\right)\right]$, so $Z \backslash S$ is a set of additive class $\eta+\bar{\eta}$ in $Z$. This terminates the proof.

\section{REFERENCES}

1. J. P. Burgess, Careful choices: $A$ last word on selectors, preprint, 1979.

2. F. Hausdorff, Über innere Abbildungen, Fund. Math. 23 (1934), 279-291.

3. L. Keldysh, On open images of A-sets, Dokl. Akad. Nauk SSSR 49 (1945), 646.

4. K. Kuratowski, Topology, Vol. 1, 5th ed., Academic Press, New York, 1966.

5. S. Levi, On the Baire property in separable metric spaces, Atti Accad. Naz. Lincei 69 (1980), 303-307.

6. A. Maitra, Selectors for Borel sets with large sections, Proc. Amer. Math. Soc. 89 (1983), 705-708.

7. D. E. Miller, Borel selectors for separated quotients, Pacific J. Math. 91 (1980), 187-198.

8. S. M. Srivastava, Selection theorems for $G_{\delta}$-valued multifunctions, Trans. Amer. Math. Soc. 254 (1979), 283-293.

9. R. L. Vaught, Invariant sets in logic and topology, Fund. Math. 82 (1974), 269-294.

Istituto di Matematica, Università di PisA, PisA, Italy 94720

Department of Statistics, UNIVERSity of CALIFORNiA, BERKELEy, CALIForNiA

Indian STATISTICAL InSTITUTE, CALCUTTA, INDiA 